\title{
The Approach to the Difficult Urethral Catheterization among Urology Residents in the United States
}

\author{
Carlos Villanueva, George P. Hemstreet III
}

Section of Urology, University of Nebraska Medical Center, Omaha, Nebraska, USA

\begin{abstract}
Purpose: To determine the prevalence of different approaches to the difficult urethral catheterization (DUC) among urology residents (UR) in the United States (US).

Materials and Methods: An email invitation to participate in an online survey regarding DUC was sent to 267 UR and to 22 urology program coordinators for them to forward to their residents. 142 UR completed the survey.

Results: After the initial unsuccessful attempt by a nurse, $92 \%$ of UR attempted a catheter prior to resorting to other modalities. The most common choice of the first catheter was a Coude (76\%) size 18F (51\%). For situations where multiple sizes and types of catheters (12 - 20F) were used without success, 3 scenarios were proposed: 1) Catheter passed the bulbomembranous urethra (BMU) and patient had previous history of transurethral resection of the prostate or radical retropubic prostatectomy, 2) Catheter passed the BMU and no urologic history, 3) Catheter did not pass the BMU and no urologic history. Flexible cystoscopy was used in 74\%, 62\% and 63\%; blind passage of a glidewire was second with 15\%, $23 \%$ and 20\%; and blind use of filiforms and followers was chosen in 7\%, $9 \%$ and $9 \%$ of the scenarios respectively.

Conclusions: The most common approach to the DUC among UR in the US involves using an 18F Coude catheter first. After trying one or more urethral catheters, UR most commonly resort to flexible cystoscopy as opposed to the blind placement of glide wires or filiforms/followers.
\end{abstract}

Key words: urethra; male; catheterization; difficult; stenosis

Int Braz, J Urol. 2010; 36: 710-7

\section{INTRODUCTION}

The difficult urethral catheterization (DUC) is one of the most common consultations for the general urologist. There are many causes of DUC among which urethral strictures and bladder neck contractures are probably the most commonly reported in the literature (Figure-1). Other less common causes include benign prostatic hyperplasia (BPH), incorrect technique, tightening of the external sphincter (in an anxious patient), false passages, phimosis, meatal stenosis, prostate cancer, etc.
There are multiple approaches to the DUC and an extensive review of the literature regarding these was recently reported (1). Common approaches include trying different sizes/types of catheters, the Liss maneuver (2), catheter guides using lidocaine jelly, filiforms/followers, blind passage of a glidewire (3), flexible cystoscopy to place a glidewire (4), suprapubic catheter, etc.

Traditionally filiforms/followers and catheter guides were the main invasive devices used by 


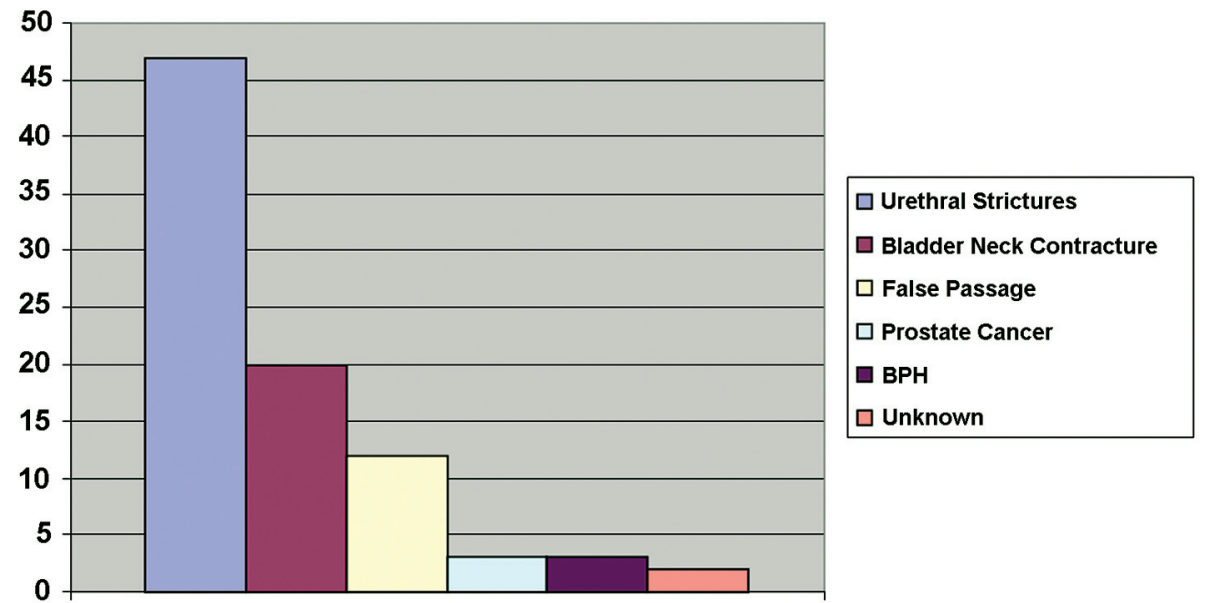

Figure 1 - Most common causes of difficult urethral catheterization*.

* Pooled cases from Beaghler's et al. (4), Freid's et al. (6) and Mistry's et al. (12) series. Included are the 54 patient from Beaghler's series all of which underwent flexible cystoscopy, and the 13 patient in Mistry's series that underwent flexible cystoscopy. Twenty patients from Freid's series were also included, but it was not mentioned in the article how the cause of difficult urethral catheterization was found in these patients.

urologists to assist with the DUC. The introduction of the flexible cystoscope and glidewires came to revolutionize the approach to the DUC. The teaching of the use of filiforms and followers is difficult, since a "feeling" has to be developed by the trainee and there is no way for the teacher to know what the trainee is feeling. Teaching flexible cystoscopy is easy in the sense that the instructor knows what is happening. This is analogous to the teaching of the use of forceps in obstetrics: any obstetrician would agree that it is easier to teach how to do a C-Section. Just as there has been a trend in obstetrics to abandon the use of forceps, there has also been a trend in urology to abandon the use of filiforms and followers. The prevalence of the various different approaches for the DUC among urologists is not known. In this study, our objective was elucidating which approaches are used by UR in the US for the DUC.

\section{MATERIALS AND METHODS}

Approval from the Institutional Review Board was granted. An email invitation was sent to 267 UR and to 22 urology residency program coordinators to forward to their residents. These residents and program coordinators were the ones we could obtain their email addresses. The email invitation contained a link for an online survey which was performed using www.surveymonkey.com. The front page of the online survey contained an informed consent.

The survey was designed to simulate common scenarios encountered during DUC. Demographic data comprised the first section: Post-graduate year (PGY) of training, state of the residency program, and the incidence of DUC encountered by the residents.

In the first case scenario, the residents were asked if they would attempt to catheterize a patient after a nurse had tried using Coude type catheters and lidocaine jelly. If they chose to try a catheter they were asked about the type and sizes.

In the second case they were asked about a scenario where during the initial attempt at catheterization with a $16 \mathrm{~F}$ Coude they felt the catheter met resistance after passing the bulbomembranous urethra (BMU). The third scenario was similar except that the catheter met resistance before the BMU. In each of these scenarios they were given the option to try one or more catheters or to proceed with cystoscopy, glidewires, filiforms or other alternatives. 
Finally they were given 3 scenarios where multiple catheter sizes and types were tried without success. In the first one the patient had a history of transurethral resection of the prostate/radical retropubic prostatectomy and the catheter on the previous attempts appeared to have met resistance past the BMU. In the second case the patient had no urologic history and the catheter on the previous attempts appeared to have met resistance past the BMU. In the last scenario the patient had no urologic history and the catheter on the previous attempts appeared to have met resistance before the BMU. In these scenarios they were given the option of initially using filiforms/followers, flexible cystoscopy, pass a glidewire blindly, suprapubic tube placement, or other.

The survey was piloted with 4 UR for ease of understanding and completion with modifications made to it accordingly. Cognitive testing was performed by having 2 residents think aloud while they were answering the questionnaire.

An internet calculator (www.surveysystem. com/sscalc.htm) was used to calculate a confidence interval (CI) based on a $95 \%$ confidence level.

\section{RESULTS}

There are probably 900 UR in the US. Our survey was completed by about $14 \%$ of all the UR if we do not count the UR doing preliminary surgery. To calculate a CI we used 900 as the population size, 127 as the sample size, $95 \%$ as the confidence level, and the worst case percentage (50\%). The CI was 8 .

The post-graduate year (PGY) distribution is depicted in Figure-2. Residents that responded to the survey were training in 27 different states. Figure-3 shows the frequency which with residents dealt with DUC in a week.

In the first scenario where a nurse had unsuccessfully tried using Coude type catheters and lidocaine jelly, $131(92 \%)$ of residents attempted to place the catheter themselves first, instead of having the nurse try an alternative approach or going directly to more advanced techniques. In this scenario, out of the $92 \%$ electing to try the catheter, the choice of the first catheter type was a Coude $(76 \%)$ with a size distribution of $18 \mathrm{~F}(51 \%), 16 \mathrm{~F}(27 \%)$ or $20 \mathrm{~F}(14 \%)$. A second catheter was selected by $97(68 \%)$ of residents, $84 \%$ chose Coude, with the sizes varying from $18 \mathrm{~F}$ $(27 \%), 20 \mathrm{~F}(25 \%), 16 \mathrm{~F}(18 \%), 12 \mathrm{~F}(14 \%)$ and $14 \mathrm{~F}$ (11\%). Only $24 \%$ of residents chose a 3 rd catheter, and only $5 \%$ a fourth.

In the next scenario, the residents were asked what to do when the initial attempt to pass a $16 \mathrm{~F}$ Coude was unsuccessful and they felt the catheter passed the BMU. In this case $63 \%$ elected to try a different catheter, $16 \%$ would go directly to flexible cystoscopy, $11 \%$ would try a glidewire blindly and $4 \%$ would use filiforms and followers. Among the 90 residents who chose to try another catheter, $87 \%$ chose a Coude, and the majority chose a larger size: $18 \mathrm{~F}(47 \%), 20 \mathrm{~F}(22 \%)$ and 22F (10\%).

In the next (similar) scenario, they were asked about what to do when the initial attempt to pass a $16 \mathrm{~F}$ Coude was unsuccessful and they felt the catheter did not pass the BMU. Only $32 \%$ elected to try a different catheter, 39\% would go directly to flexible cystoscopy, $18 \%$ would try a glidewire blindly and $8 \%$ would use filiforms and followers. Among the 45 residents that elected to try another catheter, Coude was only chosen by $40 \%$ and the majority chose a smaller catheter: $14 \mathrm{~F}(33 \%)$ and $12 \mathrm{~F}(31 \%)$.

For situations where multiple sizes and types of catheters $(12-20 \mathrm{~F})$ were used without success, 3 scenarios were proposed: 1) Catheter passed the BMU and patient had previous history of transurethral resection of the prostate or radical retropubic prostatectomy, 2) Catheter passed the BMU and no urologic history, 3) Catheter did not pass the BMU and no urologic history.

Flexible cystoscopy was used in $74 \%, 62 \%$ and $63 \%$; blind passage of a glidewire was second with $15 \%, 23 \%$ and $20 \%$; and blind use of filiforms and followers was chosen in $7 \%, 9 \%$ and $9 \%$ of the scenarios respectively. Suprapubic catheter and "other" accounted for the rest of the responses. A suprapubic catheter was used in 2.1, 0.7 and $3.5 \%$ of the scenarios respectively.

\section{COMMENTS}

Our findings confirm the observed trend of a decrease use of filiforms and followers with the 


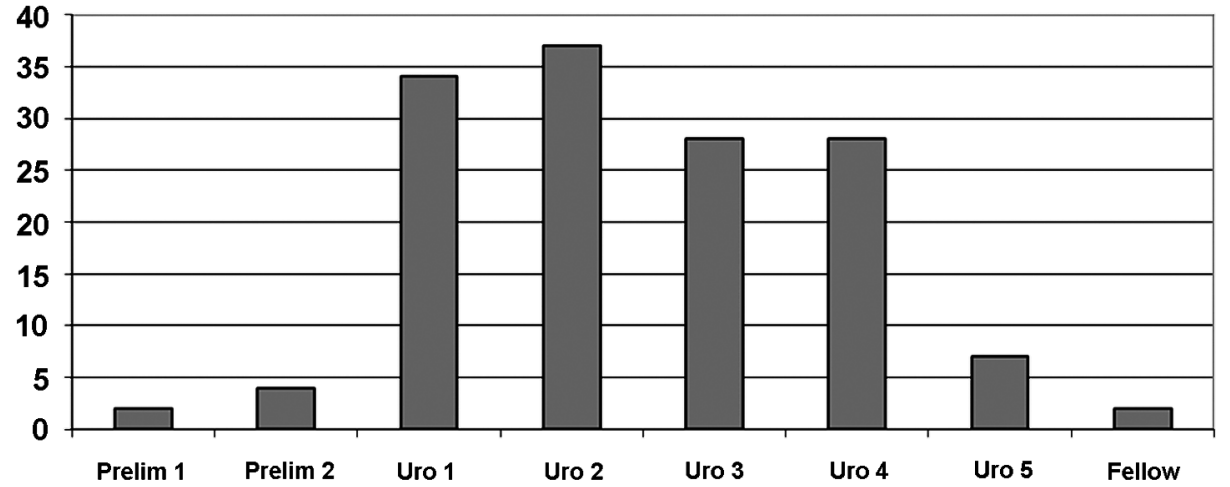

Figure 2-Post-graduate year of residents that completed the survey.

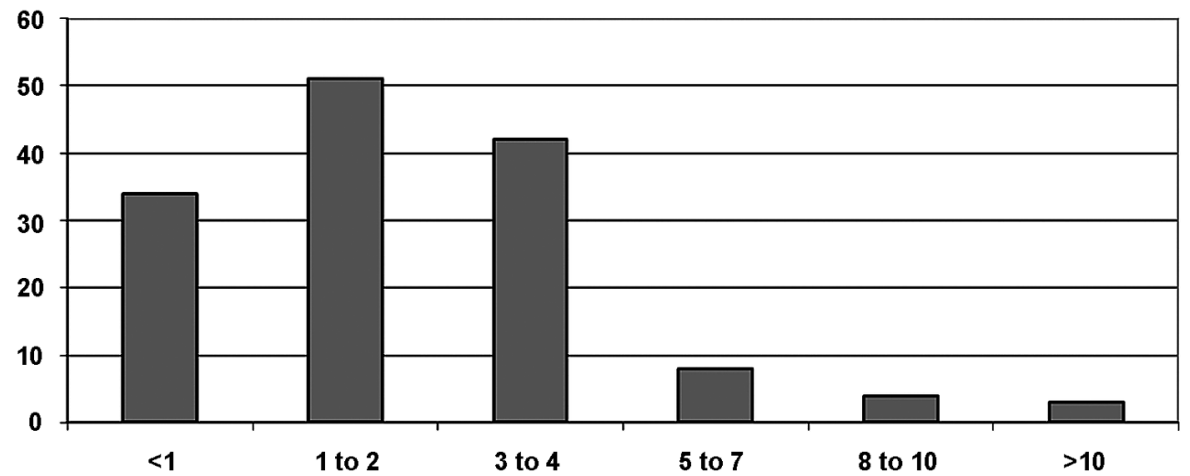

Figure 3 - Difficult urethral catheterization cases that responding residents dealt with in a week.

concomitant preference for flexible cystoscopy by UR in the US to approach the DUC. Most university hospitals nowadays have easy access to "urology carts" equipped with a flexible cystoscope. Flexible cystoscopy facilitates the diagnosis of the problem and assists maneuvering false passages and the passage of glidewires though pinpoint urethral strictures. By virtue of using a monitor, junior residents can be guided through the procedure facilitating its teaching.

Despite all the benefits of using flexible cystoscopy as the main approach to the DUC, it has not been proven that is necessary in the majority of cases. The authors have implemented a DUC algorithm (Figure-4) for all DUC cases over the past almost 3 years (report accepted for publication). This algorithm was applied to 41 patients in a University setting and 24 in the private settings successfully and without breaks. Flexible cystoscopy was required in $5 \%$ of the university patients and $13 \%$ of the private patients. No patients suffered any complications. Most urologists would agree that flexible cystoscopy is the safest approach and we agree. However, our contention is that by using algorithms like the one we reported, flexible cystoscopy could be used more selectively without harming the patient with possible (but unproven) economic and time savings.

Another prevalent approach to the DUC that the study documented among UR, was the blind pas- 


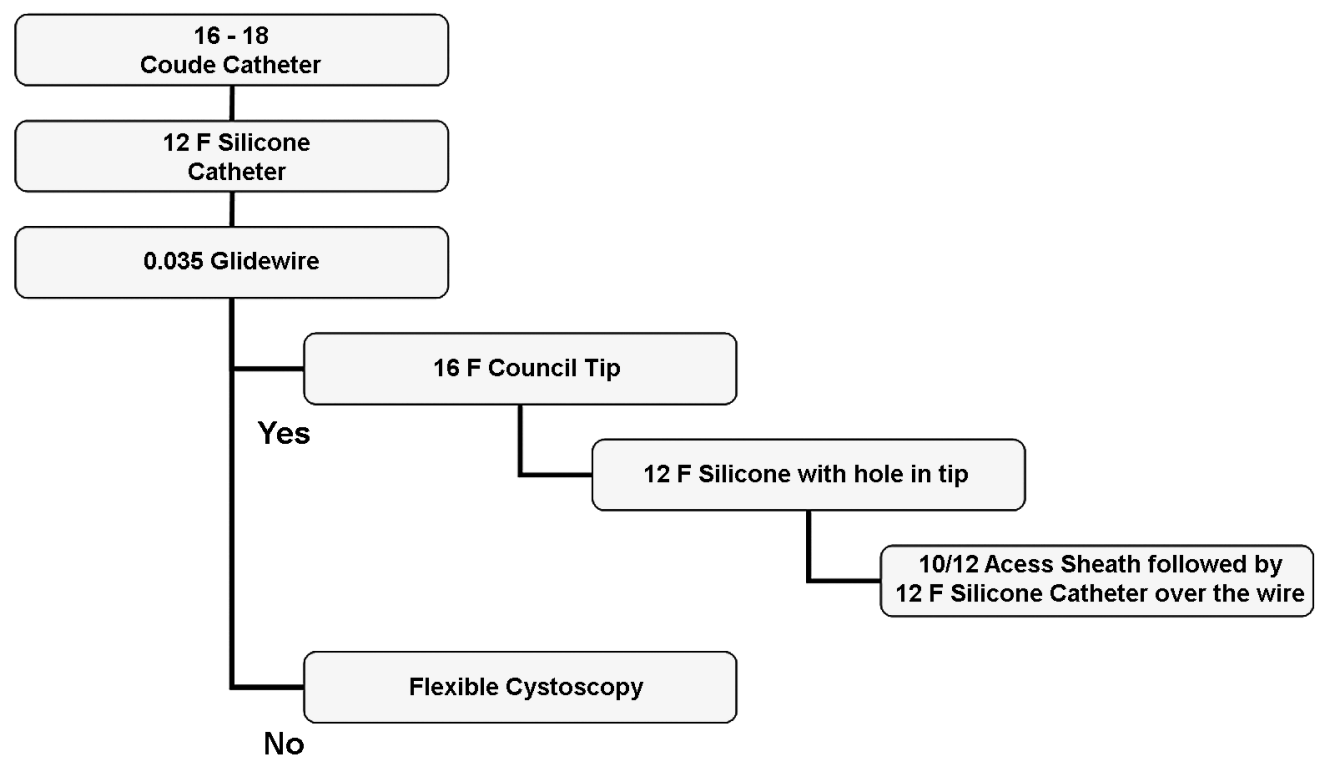

Figure 4 - Difficult urethral catheterization algorithm.

sage of a glidewire. The hydrophilic nature and floppy tips of glidewires allow them to be easily advanced into the bladder with minimal trauma. When the glidewire encounters a false passage it usually reflects out the urethral meatus instead of digging deeper into the false passage (unless a rigid shaft glidewire was used). Multiple reports have documented the safety of this approach for the DUC(3,5-7).

It is difficult to know with this survey the true incidence of the use of suprapubic catheters in the DUC. Although just a small percentage of UR chose to use a suprapubic catheter in the questionnaire, we believe that others would have also chosen it, after evaluating the situation with the flexible cystoscope. Unfortunately this was not assessed.

Although this study assesses just the initial approach to the DUC, we believe that what happens next is of paramount importance. What do residents do once a glidewire is in the bladder, whether it was passed blindly or using a flexible cystoscope, can result in severe urethral damage. In certain instances, Heyman dilators have been passed over a glidewire perforating the urethra and violating the rectum. Once a glidewire is the bladder, there are multitude choices for the next step. One can pass a small catheter without dilating or a large catheter after dilating. One can use Heyman dilators, ureteral dilators (8), ureteral access sheaths (9), urethral balloon dilators etc. It would be interesting to determine the prevalence of these different approaches among urologists.

The main weakness of the study is in the elaboration of the questionnaire. Despite the fact that our questionnaire underwent pilot testing for ease of understanding and completion as well as cognitive testing a formal validation was not performed.

Our study confirms findings analogous to those in the field of obstetrics with forceps deliveries, UR are probably not being exposed adequately to the use of the traditional filiforms and followers and will probably lack the skills to use them safely once in practice. This is compounded by the lack of studies related to the use of filiforms and followers in the DUC, with only one report briefly describing their use (10). More studies have now been published regarding the flexible cystoscopy $(4,11)$ or blind passage of glidewire approaches. A similar survey applied to practicing urologists would help assess the trend of filiforms and followers use and probably predict the future demise of one of the staple urologic instruments. 


\section{CONCLUSION}

The most common approach to the DUC among UR in the US involves using an 18F Coude catheter first. When UR attempt catheterization unsuccessfully with a $16 \mathrm{~F}$ Coude but felt the catheter was past the BMU, they chose another larger Coude catheter. When the resistance was felt before the BMU, only about a third of residents attempted another catheter, usually smaller (12 - 14F). After one or more urethral catheters, UR most commonly resort to flexible cystoscopy.

\section{CONFLICT OF INTEREST}

None declared.

\section{REFERENCES}

1. Villanueva C, Hemstreet GP 3rd: Difficult male urethral catheterization: a review of different approaches. Int Braz J Urol. 2008; 34: 401-11; discussion 412.

2. Liss MA, Leifer S, Sakakine G, Esparza M, Clayman RV: The Liss maneuver: a nonendoscopic technique for difficult Foley catheterization. J Endourol. 2009; 23: $1227-30$.

3. Zammit PA, German K: The difficult urethral catheterization: use of a hydrophilic guidewire. BJU Int. 2004; 93: 883-4.
4. Beaghler M, Grasso M 3rd, Loisides P: Inability to pass a urethral catheter: the bedside role of the flexible cystoscope. Urology. 1994; 44: 268-70.

5. Lachat ML, Moehrlen U, Bruetsch HP, Vogt PR: The Seldinger technique for difficult transurethral catheterization: a gentle alternative to suprapubic puncture. $\mathrm{Br}$ J Surg. 2000; 87: 1729-30.

6. Freid RM, Smith AD: The Glidewire technique for overcoming urethral obstruction. J Urol. 1996; 156: 164-5.

7. Chiou RK, Aggarwal H, Chen W: No title available. Can Urol Assoc J. 2009; 3: 189-192.

8. Chelladurai AJ, Srirangam SJ, Blades RA: A novel technique to aid urethral catheterisation in patients presenting with acute urinary retention due to urethral stricture disease. Ann R Coll Surg Engl. 2008; 90: 778.

9. Athanasopoulos A, Liatsikos EN: The use of a ureteral access sheath for the urethral dilatation and catheterization of difficult urethral strictures. Urol Int. 2009; 83: 359-61.

10. Jordan GH, Winslow BH, Devine CJ Jr: Intraoperative consultation for the urethra. Urol Clin North Am. 1985; 12: 447-52.

11. Krikler SJ: Flexible urethroscopy: use in difficult male catheterisation. Ann R Coll Surg Engl. 1989; 71: 3.

12. Mistry S, Goldfarb D, Roth DR: Use of hydrophiliccoated urethral catheters in management of acute urinary retention. Urology. 2007; 70: 25-7.

\section{Correspondence adrress:}

Dr. Carlos Villanueva

855 N 82nd, Plaza Apt. 40

Omaha, NE, 68114, USA

Fax: + 1402 559-6529

E-mail: cvillanueva.uro@gmail.com 


\section{EDITORIAL COMMENT}

The authors report the outcome of a questionnaire-based survey of urology residents' hypothetical preferences when dealing with difficult urethral catheterization (DUC) (1). It is estimated that the survey canvassed the opinion of approximately $14 \%$ of the current United States urological resident population. Though DUC represents a commonly encountered urological dilemma, it is largely an evidence-free issue, and the authors ought to be commended for their attempt to clarify contemporary practice and propose an algorithm. The authors claim that the algorithm has been successfully utilized in 65 consecutive patients with DUC without any failures. One arm of the algorithm proposes the passage of a hydrophilic glidewire with a catheter or access sheath passed over the wire. The alternative arm employs the use of a flexible cystoscopy. The cost of up to three catheters and an access sheath is not insignificant and may not stack up favorably against that of a multiple use flexible cystoscope. This needs to be further investigated.

Common conditions predisposing to DUC include bladder neck contractures, occlusive prostates, and urethral strictures. It is entirely appropriate for an experienced urological resident or urologist to attempt a catheterization after a failed initial attempt by a nurse. The level of obstruction, though a rather subjective entity, may suggest a particular etiology. A catheter not passing the bulbomembranous urethra in a young male with no previous urological intervention is highly suggestive of urethral stricture and multiple attempts at catheterization are to be avoided.

The use of traditional filiforms and followers is diminishing, especially in developed countries, due to a combination of lack of exposure to such techniques and the easy availability of alternative equipment such as flexible cystoscopes. Clinicians therefore not trained in these techniques may not have the 'feel' for these and are liable to cause further urethral trauma.

The use of a hydrophilic glidewire is the step suggested by the authors after two failed catheterizations. It is safer with regards to urethral trauma with the glidewire's tendency to turn back on itself rather than perforating the urethra, but in our experience, successful passage of a glidewire does not always result in successful placement of a catheter, especially in the case of dense urethral structures, and multiple attempts at 'rail-roading' a catheter over a glidewire could result in urethral trauma.

Surprisingly, there is very limited use of the suprapubic catheter (SPC) technique amongst urology residents with only $3.5 \%$ considering it a viable option. The study does not assess the reason for this and SPC does not feature at all in the proposed algorithm. SPC has inherent advantages in the management of DUC. It avoids further urethral trauma, is associated with fewer infections and urethral strictures, is more comfortable, easier to manage and more cost effective, and avoids the need for re-catheterization in the event of subsequent failure to void (2).

Note also that catheterizing a patient with a DUC will result in rapid resolution of painful retention, but does not complete the management of the patient. More often than not the patient will require a subsequent cystoscopy (flexible or rigid) to confirm urethral patency and correction of the underlying problem. This is another argument in favor of using the flexible cystoscopy to assist initial catheterization as the cause of the obstruction can be easily identified, safely negotiated and the appropriate definitive treatment can be planned.

Ultimately it is difficult to draw robust conclusions from a hypothetical survey prone to bias, but the proposed algorithm represents a logical approach to the management of DUC. In reality, local practice will be dictated by a large number of variables including local expertise, availability of equipment (e.g. dilators, flexible cystoscopes), confidence to perform SPC, and patient co-morbidity. Each case must be managed on merit.

\section{REFERENCES}

1. Villanueva C, Hemstreet GP 3rd: The Approach to the Difficult Urethral Catheterization among Urology Residents in the United States. Int Braz J Urol. 2010; in press. 
2. Horgan AF, Prasad B, Waldron DJ, O'Sullivan DC: Acute urinary retention. Comparison of suprapubic and urethral catheterisation. Br J Urol. 1992; 70: 14951.

Dr. Shalom J. Srirangam

Department of Urology

East Lancashire Teaching Hospitals NHS Trust

Preston Royal Infirmary

Preston, United Kingdom

E-mail:sjsrirangam@yahoo.co.uk

\section{REPLY BY THE AUTHORS}

We appreciate the kind comments. With regards to the algorithm that we have used in 65 consecutive patients, an access sheath was only used in 3 and cystoscopy was only necessary in 5 patients. Twenty-nine patients were catheterized readily with an $18 \mathrm{~F}$ code, and another 10 patients with the $12 \mathrm{~F}$ silicone catheter. If we had resorted to flexible cystoscopy in all of these patients, the costs would certainly have been higher. We do agree that flexible cystoscopy could have provided some useful information in the patients with pathology. Nevertheless, in our series a significant proportion of patients did not have any consequential pathology, based on the fact that the 18F Coude was successful in 29 of the 65 patients.

In our experience, we have almost always been able to place a catheter after a glidewire is secured in the bladder. In the case of a dense stricture, using a $15 \mathrm{~F}$ ureteral balloon dilator followed by a $12 \mathrm{~F}$ silicone catheter over the glidewire usually is effective. We do agree that SPC is underutilized and that it has all the mentioned advantages. As to why it is underutilized, we think It may be attributed to the setting, with most cases of DUC being performed on awake patients under no sedation many of them on anticoagulation. Also, residents most likely would have to check with their faculty when a SPC is planned whereas they will not probably do so for performing flexible cystoscopy at the bedside. This is, of course, all hypothetical and it would be interesting to study the factors that contribute to the low incidence of SPC in the setting of the DUC.

The Authors 